\title{
$\beta$-decay studies of ${ }^{135-137}$ Sn using selective resonance laser ionization techniques
}

\author{
J. Shergur, ${ }^{1}$ B. A. Brown, ${ }^{2}$ V. Fedoseyev, ${ }^{3}$ U. Köster, ${ }^{4}$ K.-L. Kratz, ${ }^{5}$ D. Seweryniak, ${ }^{1,6}$ W. B. Walters, ${ }^{1}$ A. Wöhr, ${ }^{1}$ \\ D. Fedorov, ${ }^{7}$ M. Hannawald, ${ }^{5}$ M. Hjorth-Jensen, ${ }^{8}$ V. Mishin, ${ }^{3}$ B. Pfeiffer, ${ }^{5}$ J. J. Ressler, ${ }^{1}$ H. O. U. Fynbo, ${ }^{4}$ P. Hoff, ${ }^{9}$ H. Mach, ${ }^{10}$ \\ T. Nilsson, ${ }^{4}$ K. Wilhelmsen-Rolander, ${ }^{11}$ H. Simon, ${ }^{4}$ A. Bickley, ${ }^{1}$ and the ISOLDE Collaboration ${ }^{4}$ \\ ${ }^{1}$ Department of Chemistry, University of Maryland, College Park, Maryland, 20742-2021 \\ ${ }^{2}$ Department of Physics and Astronomy and National Superconducting Cyclotron Laboratory, Michigan State University, \\ East Lansing, Michigan 48824-1321 \\ ${ }^{3}$ Institute of Spectroscopy, Russian Academy of Sciences, RU-142092 Troitsk, Russia \\ ${ }^{4}$ Experimental Physics Division, ISOLDE, CERN, CH-1211 Geneva 23, Switzerland \\ ${ }^{5}$ Institut für Kernchemie, Universität Mainz, D-55099 Mainz, Germany \\ ${ }^{6}$ Physics Division, Argonne National Laboratory, Argonne, Illinois 60439 \\ ${ }^{7}$ Petersburg Nuclear Physics Institute, RAS 188350, Gatchina, Russia \\ ${ }^{8}$ Department of Physics, University of Oslo, NO-0316 Oslo, Norway \\ ${ }^{9}$ Department of Chemistry, University of Oslo, NO-1163 Oslo, Norway \\ ${ }^{10}$ Department of Neutron Research, Uppsala University, S-61182 Nyköping, Sweden \\ ${ }^{11}$ Department of Physics, Stockholms Universitet, S-11385 Stockholm, Sweden
}

(Received 30 June 2001; published 22 February 2002)

\begin{abstract}
The decays of the very neutron rich Sn isotopes ${ }^{135-137}$ Sn were studied at CERN/ISOLDE using isotopic and isobaric selectivity achieved by the use of a resonance ionization laser ion source and mass spectroscopy, respectively. Neutron decay rates, $\gamma$-ray singles, and $\gamma$ - $\gamma$ coincidence data were collected as a function of time. The half-life $\left(T_{1 / 2}\right)$ and delayed neutron emission probability $\left(P_{n}\right)$ values of ${ }^{135} \mathrm{Sn}$ were measured to be $530(20) \mathrm{ms}$ and $21(3) \%$, respectively. For ${ }^{136} \mathrm{Sn}$, a $T_{1 / 2}$ of $250(30) \mathrm{ms}$ was determined along with a $P_{n}$ value of $30(5) \%$. For ${ }^{137} \mathrm{Sn}$, a $T_{1 / 2}$ of $190(60) \mathrm{ms}$ and a $P_{n}$ value of 58(15)\% were deduced. Identification of low-energy transitions in ${ }^{135} \mathrm{Sb}$ was made possible by comparison of laser-on and laser-off $\gamma$-ray spectra. Those data combined with $\gamma-\gamma$ coincidence spectra were used to construct a level scheme for ${ }^{135} \mathrm{Sb}$ that includes an unexpectedly low first excited state at $282 \mathrm{keV}$. A ground state $\beta$ branch of $33.2 \%$ was measured by following the growth and decay of the ${ }^{135} \mathrm{Sb}$ daughter. Shell-model calculations are consistent with the observed ${ }^{135} \mathrm{Sb}$ level structure and can account for a lowered first excited state.
\end{abstract}

DOI: 10.1103/PhysRevC.65.034313 PACS number(s): 23.40. $-\mathrm{s}, 29.30 .-\mathrm{h}, 26.30 .+\mathrm{k}, 27.60 .+\mathrm{j}$

\section{INTRODUCTION}

The structure and decay properties of the neutron-rich nuclides near double-magic ${ }^{132} \mathrm{Sn}$ are of interest to both astrophysicists and nuclear physicists. For astrophysicists, models for relating the large $r$-process abundance peaks at $A=130$ and $A=195$ to the properties of the $r$-process itself are dependent on the proper identification of waiting-point nuclides, most of which are not accessible experimentally $[1,2]$. For nuclear scientists, comparison of the level structures of these nuclides that have $N / Z$ greater than 1.6 with the level structures of similar nuclides with $N / Z \sim 1$ provide an opportunity to develop a unified approach to nuclear structure with predictive power beyond nuclei that are not currently accessible for study. In particular, the identification of singleparticle energies in nuclei adjacent to ${ }^{132} \mathrm{Sn}$ and the evolution of those levels will provide a proper basis for shell-model calculations.

In this paper, we report in detail the results of a study of the decay of ${ }^{135-137} \mathrm{Sn}$, isolated at CERN/ISOLDE by selective laser ionization. $\beta$-delayed neutron $(\beta \mathrm{dn})$ decay rates, $\gamma$-ray singles spectra, and $\gamma-\gamma$ coincidences were collected as a function of time with the laser both on and off. Half-lives and delayed neutron branches are presented for all three nuclides, as is a decay scheme for ${ }^{135} \mathrm{Sn}$ to levels of ${ }^{134,135} \mathrm{Sb}$. A preliminary report of these data has been published by Shergur et al. [3].

\section{EXPERIMENTAL PROCEDURES AND RESULTS}

Neutron-rich nuclei were produced at CERN/ISOLDE by spallation of a $\mathrm{UC}_{2}$ target $\left(20 \mathrm{~cm}\right.$ long, thickness $52 \mathrm{~g} / \mathrm{cm}^{2}$ and about $10 \mathrm{~g} / \mathrm{cm}^{2}$ of graphite) with $1 \mathrm{GeV}$ protons that were accelerated by the Proton Synchrotron Booster (PSB). Approximately $3 \times 10^{13}$ protons strike the target in each proton pulse. PSB pulses are available in $1.2 \mathrm{~s}$ intervals. In these experiments, the minimum interval was $2.4 \mathrm{~s}$ that provides for a data collection period of $\sim 1 \mathrm{~s}$ and $\sim 1 \mathrm{~s}$ for tape movement. Data collection periods as long as $12 \mathrm{~s}$ were used to follow growth and decay of daughter activities.

Sn isotopes were selectively ionized by use of a resonance ionization laser ion source (RILIS). Previous studies at CERN/ISOLDE have shown that the use of RILIS can significantly increase the yields of specific nuclides relative to the inevitable products of surface ionization [4,5]. The ionization scheme used for $\mathrm{Sn}$ nuclei was based on three resonant transitions, $\quad\left(\lambda_{1}=300.9 \mathrm{~nm}, \lambda_{2}=811.4 \mathrm{~nm}\right.$, and $\lambda_{3}$ $\leqslant 832.5 \mathrm{~nm}$ ) leading to an autoionizing state [4]. Sn ions were extracted into the general purpose separator for mass separation and implanted into an $\mathrm{Al}$ coated tape in one of two tape stations. At one station, $\beta$-delayed neutrons were 


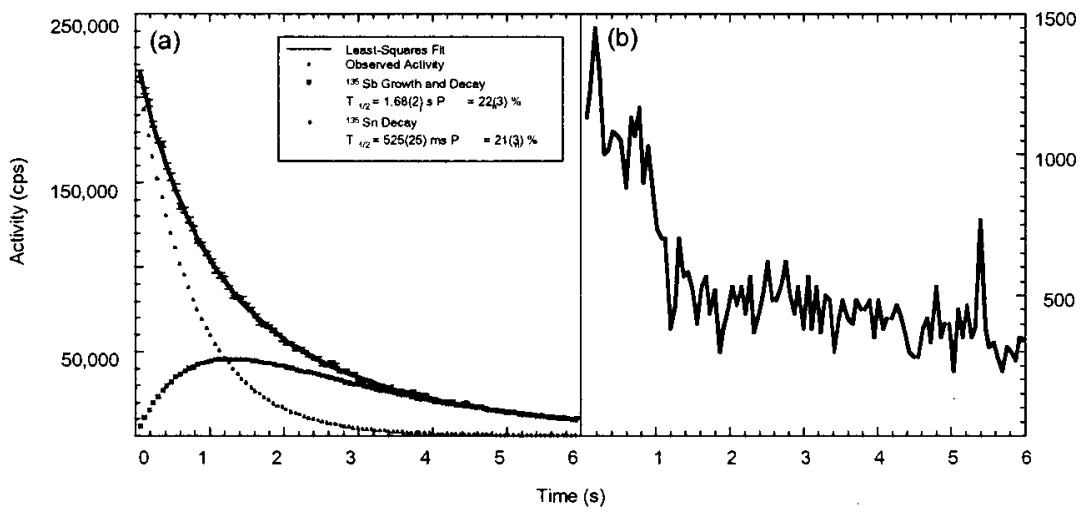

FIG. 1. Shown in (a) is a least-squares fit for the background-subtracted $\beta \mathrm{dn}$ decay of ${ }^{135} \mathrm{Sn}$. Both the decay of ${ }^{135} \mathrm{Sn}$ and growth and decay of ${ }^{135} \mathrm{Sb}$ are plotted. The least-squares fit yielded $T_{1 / 2}$ and $P_{n}$ values of 525(25) $\mathrm{ms}$ and $21(3) \%$, respectively. In (b), a $\beta \mathrm{dn}$ spectrum was obtained at $A=135$ with the laser-off. counted using the high-efficiency Mainz neutron long counter. This counter consists of a three-ring concentric array of $50{ }^{3} \mathrm{He}$ proportional counters. Neutron counts were recorded as a function of time in $1-10 \mathrm{~ms}$ bins. This station is also equipped with a thin plastic $\beta$ detector to record $\beta$-decay electrons, again, in $1-10 \mathrm{~ms}$ time bins. In both tape stations, the measurement follows implantation with the clock starting when the proton pulse hits the target. The tape is moved after each measurement period to avoid buildup of long-lived isobaric activity in front of the detectors. In both stations, data were taken with the laser-on and also with the laser-off.

$\gamma$-rays were counted at the other tape station with three orthogonal $\mathrm{Pb}$-shielded Ge detectors. For each event, the outputs from all three Ge detectors were recorded along with time-to-amplitude converter information and the time of the event after the proton pulse. From these data, it was possible to construct a $\gamma$ - $\gamma$ coincidence matrix, and a $\gamma-(t)$ matrix. Projections from these matrices were used to determine the time dependence of each $\gamma$-ray and generate coincidence spectra. These techniques have been previously described by Hannawald et al., in studies of $\mathrm{Cd}$ and Mn decays [6,7].

\section{A. ${ }^{135} \mathrm{Sn}$}

In Fig. 1 is shown the time dependence of the $\beta$-delayed neutron $(\beta \mathrm{dn})$ count rate taken with the laser-on and also

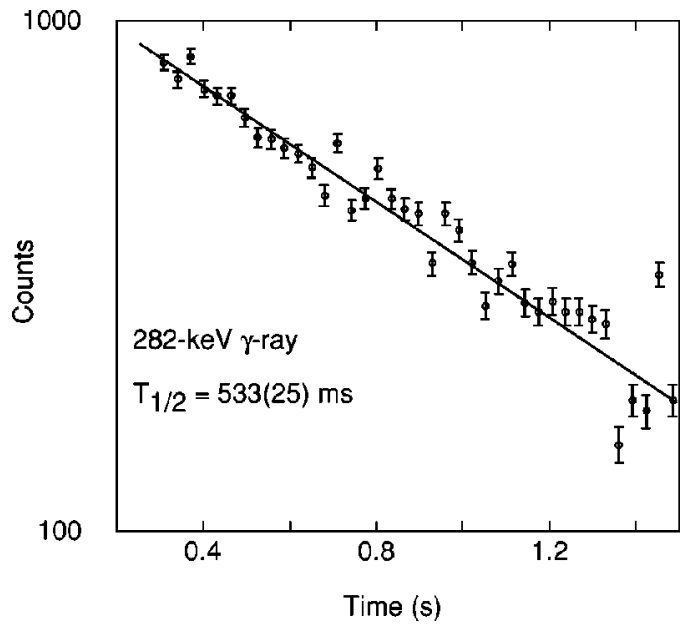

FIG. 2. Least-squares fit of the decay of the $282-\mathrm{keV}$ transition in ${ }^{135} \mathrm{Sb}$ following $\beta$-decay of ${ }^{135} \mathrm{Sn}$. with the laser-off. The laser-on spectrum shown has been corrected for the laser-off contributions. A least-squares fit for the neutrons from ${ }^{135} \mathrm{Sn}$ decay and the neutrons from the growth and decay of daughter ${ }^{135} \mathrm{Sb}$ is shown. Using values of $1.68(2) \mathrm{s}$ and $22(3) \%$ for the $T_{1 / 2}$ and $P_{n}$, respectively for ${ }^{135} \mathrm{Sb}$ [8], a value of $525(25) \mathrm{ms}$ is obtained for the half-life of ${ }^{135} \mathrm{Sn}$, the same as shown in the figure caption, along with a $P_{n}$ value of $21(3) \%$. The time spectrum projected from the $\gamma$ - $(t)$ matrix for the 282-keV $\gamma$-ray is shown in Fig. 2. Corrections have been made for Compton events that also fall in the $282-\mathrm{keV}$ energy window. A $T_{1 / 2}$ of $533(25) \mathrm{ms}$ is obtained from these data. Combining our data from the time dependence of the $\beta \mathrm{dn}$ and $\gamma$-ray data, we obtain a $T_{1 / 2}$ of $0.53(2)$ s for ${ }^{135} \mathrm{Sn}$. A $T_{1 / 2}$ measurement of $0.6(1) \mathrm{s}$ and $\gamma$ rays at 282, 317, 732, and $925 \mathrm{keV}$ have been observed in ${ }^{135} \mathrm{Sn}$ decay by Korgul et al. [9].

In Figs. 3 and $4, \gamma$-ray spectra are shown that were projected from the $\gamma-(t)$ matrix. The spectrum accumulated during the first $1.32 \mathrm{~s}$ after the start of counting is shown [Fig. 3(a), Fig. 4(a)] along with the $\gamma$-rays accumulated during the second $1.32 \mathrm{~s}$ [Fig. 3(b), Fig. 4(b)] and a third spectrum taken with the laser off [Fig. 3(c), Fig. 4(c)]. Comparison of

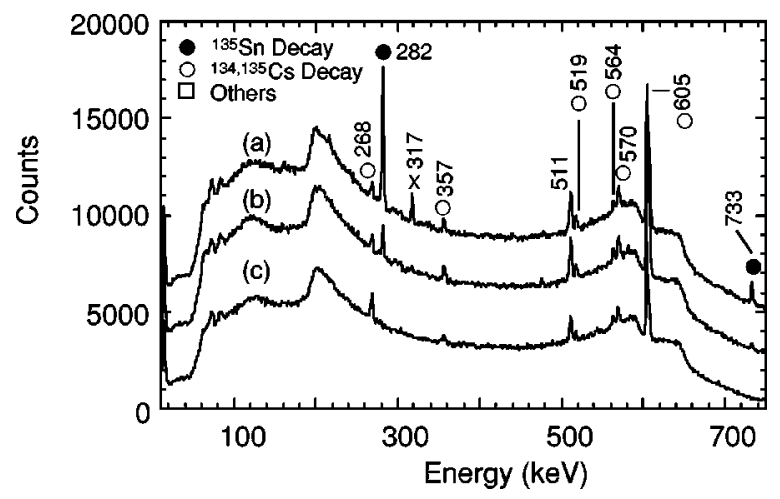

FIG. 3. $\gamma$-spectra obtained following the decay of ${ }^{135} \mathrm{Sn}$. In (a), data were obtained during the first $1.32 \mathrm{~s}$ following proton spallation of the target with the laser on. Spectrum (b) was taken in the following $1.32 \mathrm{~s}$ time interval, also with the laser on. The $\mathrm{x}$ for the $317 \mathrm{keV} \gamma$-ray designates a transition in the $\beta \mathrm{dn}$ daughter ${ }^{134} \mathrm{Sb}$. The laser-off spectrum obtained for $A=135$ is shown in (c). The peaks not assigned to the decay of ${ }^{135} \mathrm{Sn}$ can be attributed to either the decay of ${ }^{135} \mathrm{Cs}$ nuclei produced from surface-ionization in the target or decay from long-lived ${ }^{134} \mathrm{Cs}$ contamination buildup on the Al tape. 


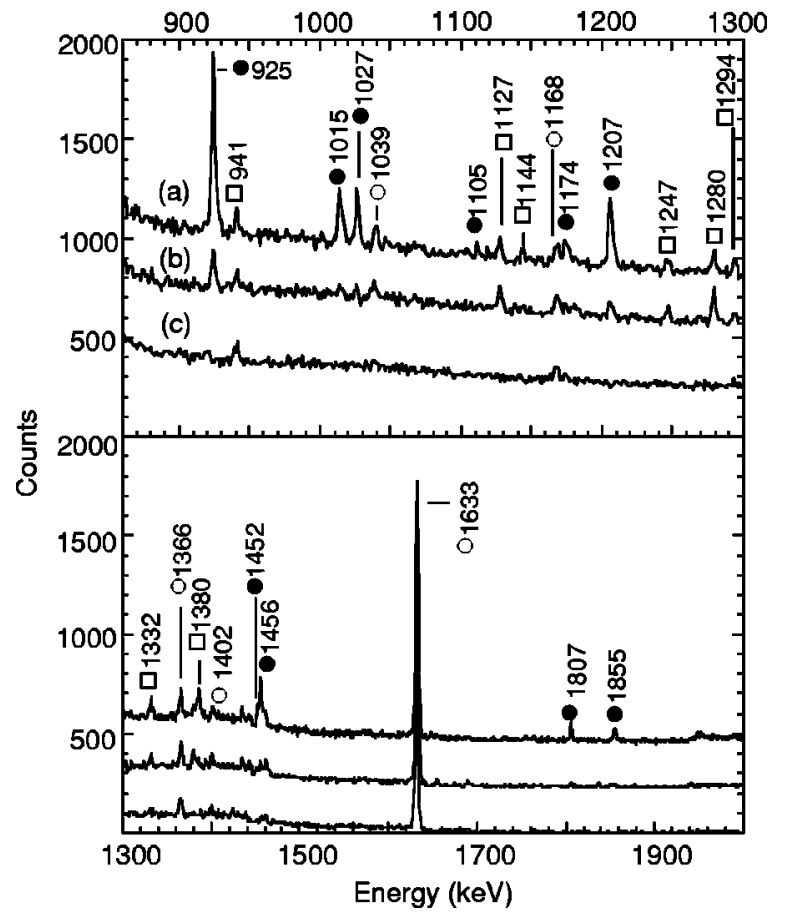

FIG. 4. Spectra (a), (b), and (c) have the same designation as in Fig. 3, and span the remaining energy range of observed transitions. Peaks not labeled with a square are attributed to the previously discussed Cs contamination or the growth of the decay daughters (i.e., ${ }^{135} \mathrm{Sb}$ ).

Fig. 3(a) and Fig. 3(b) shows the decay of the 282-, 317-, and $733-\mathrm{keV}$ transitions associated with the decay of ${ }^{135} \mathrm{Sn}$. None of these three transitions are present in the laser-off spectrum [Fig. 3(c)]. The 317-keV $\gamma$-ray has been previously identified as depopulating a low-energy state in ${ }^{134} \mathrm{Sb}[10]$. The $\gamma$-rays in the laser-off spectrum arise from long lived ${ }^{134,135} \mathrm{Cs}$ implanted on the tape.

The spectrum of $\gamma$-rays in coincidence with the $282-\mathrm{keV}$ $\gamma$-ray projected from the $\gamma-\gamma$ matrix is shown in Fig. 5. The energies, intensities, and placements of the $\gamma$-rays assigned to the levels of ${ }^{135} \mathrm{Sb}$ are presented in Table I.

The growth and decay of known ${ }^{135} \mathrm{Sb}$ decay $\gamma$-rays were used to determine the ground-state $\beta$-branching for ${ }^{135} \mathrm{Sn}$ decay. The $\gamma$-ray spectrum for ${ }^{135} \mathrm{Sb}$ decay taken around the

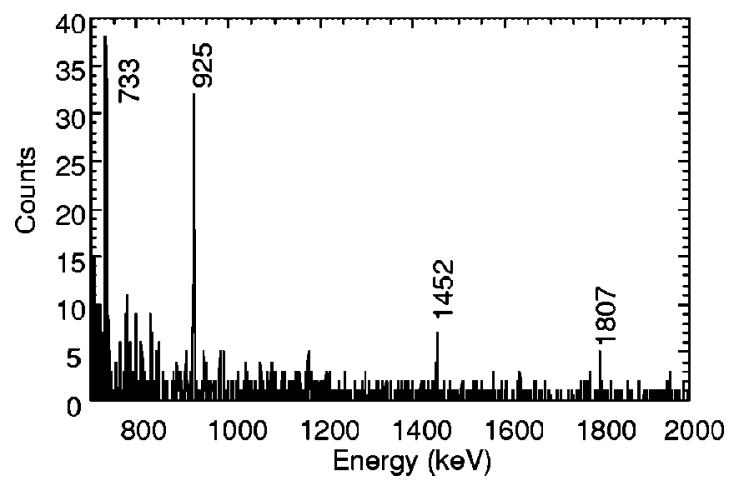

FIG. 5. $\gamma-\gamma$ coincidence spectrum gated on the $282 \mathrm{keV} \gamma$-ray transition in ${ }^{135} \mathrm{Sb}$ following $\beta$ decay of ${ }^{135} \mathrm{Sn}$.
TABLE I. Data for $\gamma$-rays and levels observed in the $\beta$-decay of ${ }^{135} \mathrm{Sn}$.

\begin{tabular}{lcc}
\hline \hline Level $(\mathrm{keV})$ & $E_{\gamma}(\mathrm{keV})$ & $I_{\gamma}{ }^{\mathrm{a}}$ \\
\hline 282 & 282 & 100 \\
1015 & 1015 & $10.8(14)$ \\
& 733 & $26(4)$ \\
1027 & 1027 & $12.3(15)$ \\
1207 & 1207 & $16.6(10)$ \\
& 925 & $33(3)$ \\
1387 & 1105 & $1.8(2)$ \\
1456 & 1456 & $7.9(5)$ \\
& 1174 & $3.3(5)$ \\
1734 & 1452 & $1.0(1)$ \\
1855 & 1855 & $3.7(4)$ \\
2089 & 1807 & $5.4(5)$ \\
\hline \hline
\end{tabular}

${ }^{a}$ Relative to the $282-\mathrm{keV}$ transition.

maximum of the ${ }^{135} \mathrm{Sb}$ activity level as observed in the delayed-neutron spectrum is shown in Fig. 6. Using the reported intensities for ${ }^{135} \mathrm{Sb}$ decay [11], the $\beta$-branches for ${ }^{135} \mathrm{Sn}$ decay are shown in the decay scheme presented in Fig. 7. These $\log f t$ values are, of course, lower limits as weak transitions populating these levels may not have been observed. However, ${ }^{135} \mathrm{Sb}$ has only three neutrons outside of double-magic ${ }^{132} \mathrm{Sn}$ and a neutron separation energy $S_{n}$ of 3.8 MeV. Because the core excitation of ${ }^{132} \mathrm{Sn}$ is $\sim 4 \mathrm{MeV}$, there are only a limited number of levels in ${ }^{135} \mathrm{Sb}$ below that energy and hence, fewer higher-energy levels to be populated and whose weak decay might have been missed.

\section{B. ${ }^{136} \mathrm{Sn}$}

In Fig. 8(a) are shown the laser-on and laser-off $\beta \mathrm{dn}$ count rates observed at $A=136$. In Fig. 9, we show the least-

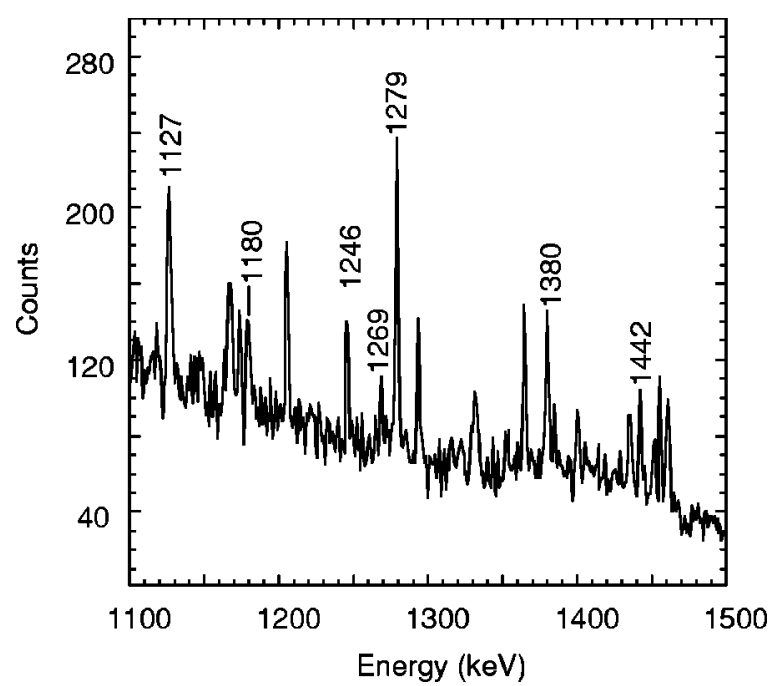

FIG. 6. $\gamma$-spectrum obtained from a $600 \mathrm{~ms}$ time slice that corresponded to the maximum buildup of ${ }^{135} \mathrm{Sb}$ growth from the $\beta$ decay of ${ }^{135} \mathrm{Sn}$. The labeled transitions are the most intense that occur in the daughter nucleus ${ }^{135} \mathrm{Te}$ following $\beta$-decay of ${ }^{135} \mathrm{Sb}$. 


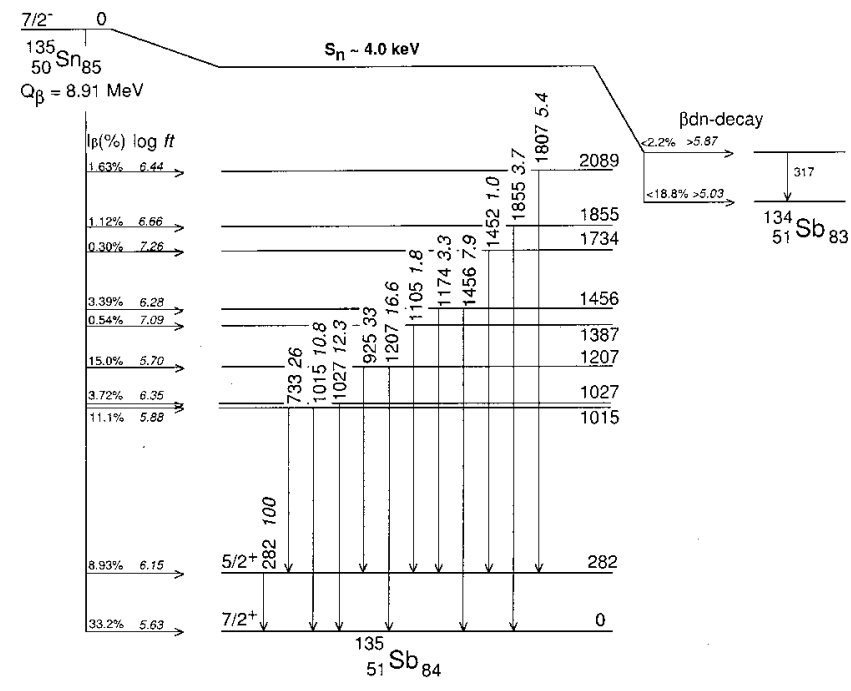

FIG. 7. Proposed decay scheme of ${ }^{135} \mathrm{Sn}$, showing the population of states in ${ }^{135} \mathrm{Sb}$ and the feeding of states in ${ }^{134} \mathrm{Sb}$ via $\beta$ delayed neutrons.

squares analysis of the laser-on background corrected data that includes the reported $T_{1 / 2}$ and $P_{n}$ values for ${ }^{136} \mathrm{Sb}$ and

${ }^{135} \mathrm{Sb}$ [8]. The fit was used to obtain $T_{1 / 2}$ and $P_{n}$ values of $250(30) \mathrm{ms}$ and 30(5)\%. No $\gamma$-rays were found in the timeprojected $\gamma$-ray spectra that exhibited a short $T_{1 / 2}$ in the 250 ms range. In particular, the $282-\mathrm{keV}$ line in ${ }^{135} \mathrm{Sb}$ that would have been populated in the $30 \% \beta \mathrm{dn}$ branch was not observed owing, in part, to the intense isobaric $\mathrm{Cs}$ and $\mathrm{Ba}$ lines present in the $\gamma$-ray spectrum. Previous structure studies of this nucleus have only yielded information regarding a 565(50) ns isomeric state whose depopulation includes a $\gamma$-ray at $173 \mathrm{keV}[12]$.

$$
\text { C. }{ }^{137} \mathrm{Sn}
$$

The $\beta \mathrm{dn}$ laser-on and laser-off spectra count rates for $A$ $=137$ are shown in Fig. 8(b). Unlike the spectra at $A=135$ and $A=136$, significant activity is observed with the laser-off that exhibits a rather short $T_{1 / 2}$. These data can be fit to a $T_{1 / 2}$ of $\sim 2.5 \mathrm{~s}$ and, after considering a number of molecular species that might arise at $A=137$, but not $A=135$ and 136 , this activity was assigned to decay of independently ionized

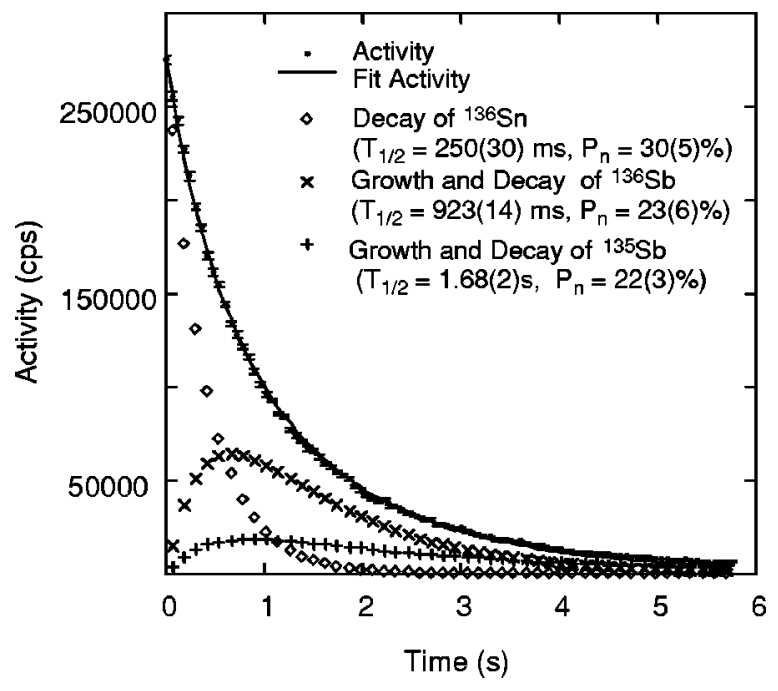

FIG. 9. Least-squares analysis for the $\beta$ dn decay of ${ }^{136} \mathrm{Sn}$.

${ }^{137}$ Te. In Fig. 10 is shown the analysis of the laser-on $A$ $=137$ neutron counting rates corrected for the laser-off activity. As there are no data available for either the $T_{1 / 2}$ or $P_{n}$ value of ${ }^{137} \mathrm{Sb}$, these fits contained four unknown quantities: the half-lives for ${ }^{137} \mathrm{Sn}$ and ${ }^{137} \mathrm{Sb}$ as well as their respective $P_{n}$ values.

An upper limit for the $T_{1 / 2}$ of ${ }^{137} \mathrm{Sn}$ can be obtained by assuming that only ${ }^{137} \mathrm{Sn}$ and $\beta \mathrm{dn}$ daughter ${ }^{136} \mathrm{Sb}$, whose $T_{1 / 2}$ and $P_{n}$ value were already noted earlier, are present (i.e., a $100 \% P_{n}$ for ${ }^{137} \mathrm{Sn}$ ). From this analysis, an upper limit of $470 \mathrm{~ms}$ is set for the $T_{1 / 2}$ of ${ }^{137} \mathrm{Sn}$. By using extrapolated values for the $T_{1 / 2}$ and $P_{n}$ value of ${ }^{137} \mathrm{Sb}$ based on the decay properties of ${ }^{133} \mathrm{Sb}$ and ${ }^{135} \mathrm{Sb}$, we can obtain $T_{1 / 2}$ estimates of $190(60)$ and $450(50) \mathrm{ms}$, respectively, for ${ }^{137} \mathrm{Sn}$ and ${ }^{137} \mathrm{Sb}$ as well as $P_{n}$ values of 58(15) and 49(10)\%, respectively. No $\gamma$-rays could be attributed to the decay of either ${ }^{137} \mathrm{Sn}$ or ${ }^{137} \mathrm{Sb}$.

\section{D. ${ }^{138} \mathrm{Sn}$}

At $A=138$, as can be seen in Fig. 8(c), there is no clear difference observed between the laser-on and laser-off time-
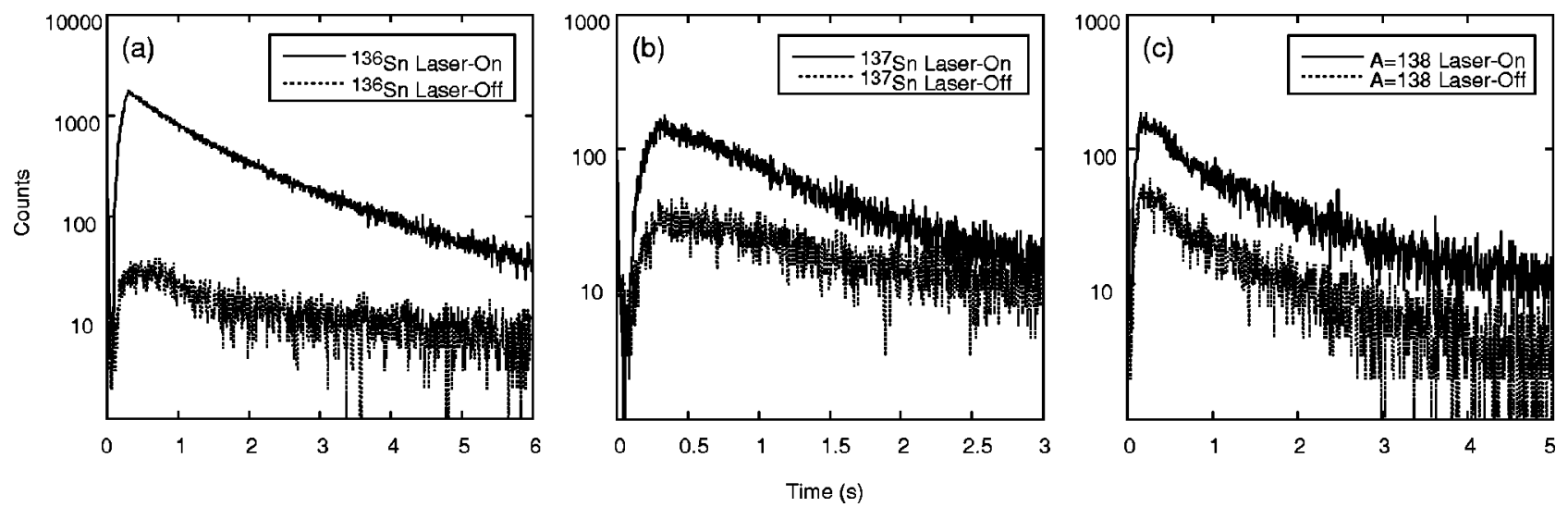

FIG. 8. Comparison of the laser-on and laser-off $\beta \operatorname{dn}$ spectra for (a) $A=136$ (b) $A=137$, and (c) $A=138$. 

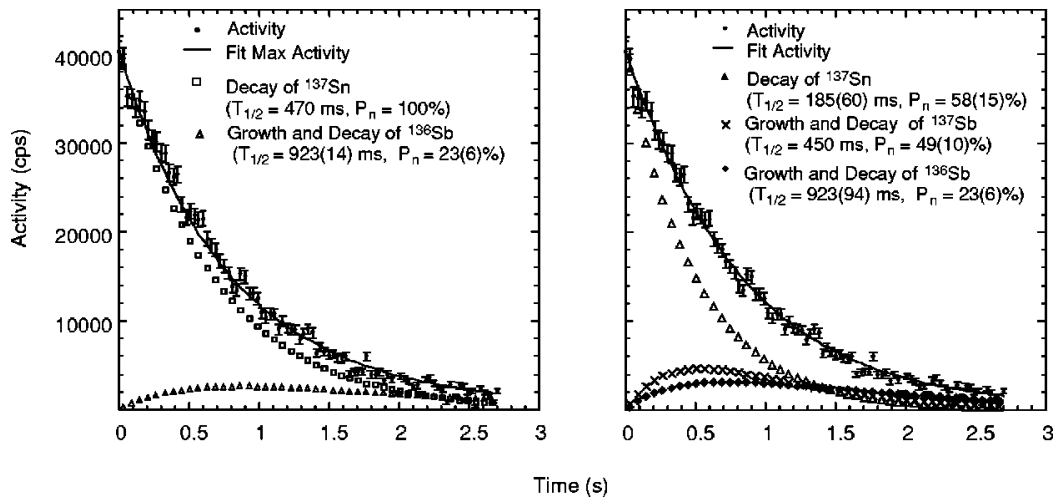

FIG. 10. In the left part, a maximum $T_{1 / 2}$ approximation for the $\beta \mathrm{dn}$ decay of ${ }^{137} \mathrm{Sn}$ was obtained by assigning the $P_{n}$ of ${ }^{137} \mathrm{Sn}$ as $100 \%$. As ${ }^{137} \mathrm{Sb}$ would not contribute to the $\beta$ dn activity under this constraint, it is possible to determine an upper limit for the $T_{1 / 2}$ of ${ }^{137} \mathrm{Sn}$. In the right part we show an optimized least-squares fit for the same $\beta$ dn spectrum. dependent neutron-decay rates. As noted for $A=137$, these neutrons can be fitted using the $T_{1 / 2}$ and $P_{n}$ values available in the literature for ${ }^{138} \mathrm{Te}$ and daughter ${ }^{138} \mathrm{I}[8]$.

\section{DISCUSSION}

\section{A. Astrophysics}

With respect to rapid neutron-capture nucleosynthesis (the astrophysical $r$-process), $\beta$-decay properties $\left(T_{1 / 2}\right.$ and $P_{n}$ values) and nuclear masses (in particular, $S_{n}$ values) of neutron-rich $\mathrm{Sn}$ isotopes heavier than double-magic ${ }^{132} \mathrm{Sn}$ are the most important nuclear-physics quantities. Under typical $r$-process conditions, even- $N$ Sn nuclides will be the classical "waiting-point" nuclei just beyond the major $A$ $\simeq 130$ solar-system $r$-abundance peak $\left(N_{r, \odot}\right.$; see, e.g., Refs. $[1,2,13])$. Odd-neutron $\mathrm{Sn}$ isotopes (with "low" $S_{n}$ values) will only build up small progenitor abundances, since they will either photodisintegrate back to an $(A-1)$ even-even isotope or capture another neutron to become an $(A+1)$ even-even $\mathrm{Sn}$ nucleus. Therefore, these even-even isotopes (here mainly ${ }^{136} \mathrm{Sn}$ and ${ }^{138} \mathrm{Sn}$ ) will be the classical " $r$-process nuclei" carrying the main $r$ abundances in the Sn isotopic chain. And only for these even-neutron isotopes is the $\beta$-decay $T_{1 / 2}$ of importance in the waiting-point concept, which implies the historical $N_{r, \odot}(Z) \times \lambda_{\beta(Z)} \simeq$ const correlation.

In Fig. 11, we show a simple static calculation of the relative $r$-abundances of neutron-rich $\mathrm{Sn}$ isotopes as a function of neutron density at freeze-out conditions $\left(T_{9}=1.35\right)$. As can be seen from this figure, at modest neutron densities of $n_{n} \simeq 1 \times 10^{23}-3 \times 10^{24} \mathrm{n} / \mathrm{cm}^{3}$ representative for the buildup of $r$ abundances in the $A \simeq 130$ peak region, ${ }^{136} \mathrm{Sn}$ will be the main waiting-point nucleus with relative abundances between $35 \%$ and $90 \%$ of the total $\mathrm{Sn}$ isotopic chain. Under these conditions, the odd-neutron neighbors ${ }^{135} \mathrm{Sn}$ and ${ }^{137} \mathrm{Sn}$ only collect roughly two orders of magnitude less $r$-progenitor yields (max. 0.5\%). For higher neutron densities in the range $n_{n} \simeq 3 \times 10^{24}-1 \times 10^{26} \mathrm{n} / \mathrm{cm}^{3}$ where the $r$-matter flow has already passed the $A \simeq 130$ "bottleneck" to form the rare-earth region and to start the climb up the staircases at the $N=126$ shell closure, ${ }^{138} \mathrm{Sn}$ becomes the main waitingpoint isotope with abundances between about $50 \%$ and $90 \%$ of the total yield.

The experimental $T_{1 / 2}$ and $P_{n}$ values can now be incorporated into dynamic (time-dependent) multicomponent $r$-process calculations to replace earlier theoretical data. A first such application (see, e.g., Fig. 3 in Ref. [1]) does improve the $r$-abundance fit in the $A \simeq 135$ region above the $A \simeq 130 N_{r} \odot$ peak; however, the still existing odd-even deviations from the $N_{r, \odot}$ values seem to indicate an earlier onset of collectivity in very neutron-rich $\mathrm{Sn}$ and Te isotopes than predicted by common mass models. This would result in slightly higher $\mathrm{S}_{n}$ values in this region, thus shifting the $r$-process path further away from $\beta$-stability involving progenitor isotopes with shorter half-lives and consequently lower final abundances.

\section{B. Nuclear Physics}

The most important nuclear-physics information concerns at $282 \mathrm{keV}$ the low energy of the first excited state of ${ }^{135} \mathrm{Sb}$, along with the redistribution of the $\beta$-decay strength. The $5 / 2^{+}$spin and parity assignments are based on systematics and the results from the high-spin states observed in fissionfragment $\gamma$-ray spectroscopy [14]. Positive parity is almost certainly indicated as the only negative parity level identified

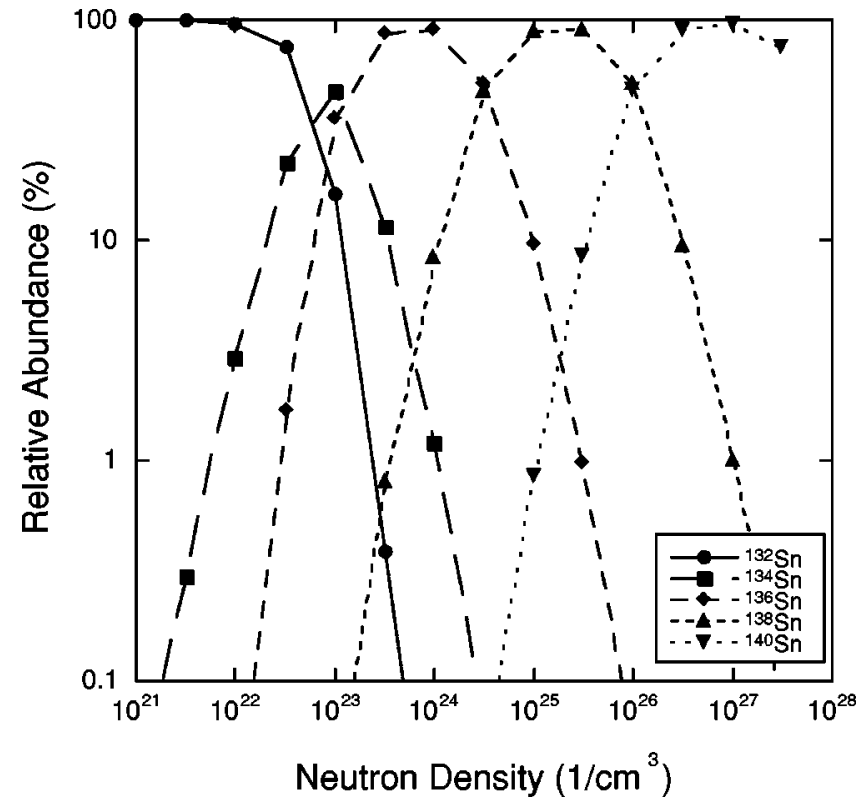

FIG. 11. Relative isotopic $r$-process abundances of $\mathrm{Sn}$ isotopes under freeze-out conditions $\left(T_{9}=1.35\right)$ as a function of neutron density. For details, see text. 
in ${ }^{133} \mathrm{Sb}$ is the $h_{11 / 2}$ level at $2763 \mathrm{keV}$ [15]. With a $\log f t$ value of 6.15 , the decay is either allowed or first forbidden, restricting the state to spin values of $5 / 2^{+}, 7 / 2^{+}$, or $9 / 2^{+}$. If this state were $9 / 2^{+}$, it certainly should have been observed strongly in the fission-fragment spectroscopy [14]. If this state were to be a $7 / 2^{+}$level, it would pose the strange situation of two low-energy $7 / 2^{+}$levels in a single nucleus, a situation rarely encountered.

The $\log f t$ value for the decay of the single $f_{7 / 2}$ neutron in ${ }^{133} \mathrm{Sn}$ to the single $g_{7 / 2}$ ground state of ${ }^{133} \mathrm{Sb}$ is 5.44 , whereas the $\log f t$ for decay to the $d_{5 / 2}$ first excited state of ${ }^{133} \mathrm{Sb}$ was significantly higher at 6.05 [15]. Using those values and a $Q$ value of $8.91 \mathrm{MeV}$ for ${ }^{135} \mathrm{Sn}$ decay, a $T_{1 / 2}$ of $300 \mathrm{~ms}$ had been estimated for ${ }^{135} \mathrm{Sn}$ prior to this experiment for use in $r$-process calculations.

In spite of having three neutrons that can undergo $\beta$-decay, the $\log f t$ value of 5.63 that we deduce for the decay of the $7 / 2^{-}$ground state in ${ }^{135} \mathrm{Sn}$ to the $g_{7 / 2}$ in ${ }^{135} \mathrm{Sb}$ is 0.19 units higher than noted above for the ${ }^{133} \mathrm{Sn}$ decay. This one-third reduction in the ground state to ground state transition rate reflects both a broader admixture of the $g_{7 / 2}$ single-particle wave function into higher-energy levels as would be expected in the daughter nucleus, as well as the $\beta$-decay channels opened up by the occupancy of other neutron orbitals by the extra pair of neutrons. Conversely, the smaller reduction in the $\beta$-decay transition rate indicated by the smaller increase in the $\log f t$ from 6.05 to 6.15 for the transition to the lowest $5 / 2^{+}$level at $282 \mathrm{keV}$ in ${ }^{135} \mathrm{Sb}$ relative to the transition to the $d_{5 / 2}$ level at $963 \mathrm{keV}$ in ${ }^{133} \mathrm{Sb}$ suggests that the $282 \mathrm{keV}$ level has retained more of its single-particle character than the $g_{7 / 2}$ ground state.

We have also calculated a minimum $\log f t$ value for the $\beta \mathrm{dn}$ feeding of the ground state in ${ }^{134} \mathrm{Sb}$, assuming that all of the delayed neutron decay populates the levels shown near the ground state of ${ }^{134} \mathrm{Sb}$. Transition strength to higher energy levels would have lower log $f t$ values. Because this value is already at 5.0 and possibly lower we interpret the decay to levels above $4 \mathrm{MeV}$ as the Gamov Teller allowed transition of an $h_{9 / 2}$ neutron (from small occupancy of a pair of neutrons in those orbitals) to an $h_{11 / 2}$ proton whose unperturbed energy in ${ }^{133} \mathrm{Sb}$ is $2793 \mathrm{keV}$. Such three-particle final states with $\pi h_{11 / 2} \nu h_{9 / 2} \nu f_{7 / 2}$ configurations would be expected to lie beyond the estimated neutron separartion energy of $\sim 4 \mathrm{MeV}$ in ${ }^{135} \mathrm{Sb}$.

The sharply lowered position of the first excited $5 / 2^{+}$ level was unexpected. The trend of the monopole increases for the $d_{5 / 2}$ orbital relative to the $g_{7 / 2}$ ground state as $N$ $=82$ is approached is shown in Fig. 12. Hence, the lowest $5 / 2^{+}$level might have been expected to lie near the $700 \mathrm{keV}$ energy of the first $2^{+}$energy of the ${ }^{134} \mathrm{Sn}$ core and have a dominant $g_{7 / 2} \times 2^{+}$configuration.

One method for highlighting the unusual behavior of this $5 / 2^{+}$level is to make comparisons of the energy gap for the lowest $5 / 2^{+}$and $7 / 2^{+}$orbitals in isotones of higher $Z$ oddproton nuclides. The energy difference between the lowest $5 / 2^{+}$level and lowest $7 / 2^{+}$level is plotted in Fig. 13 for the ${ }_{51} \mathrm{Sb},{ }_{53} \mathrm{I},{ }_{55} \mathrm{Cs}$, and ${ }_{57} \mathrm{La}$ isotones with $72 \leqslant N \leqslant 84$. Except for the $N=84$ isotones, a sharp drop in this energy difference

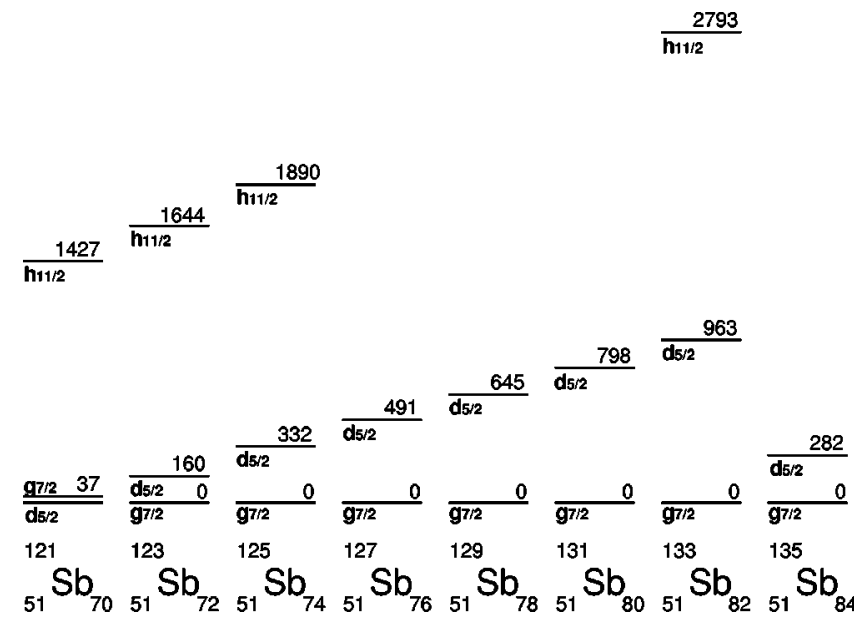

FIG. 12. Level systematics of the neutron-rich odd-mass Sb isotopes.

occurs as $Z$ increases from 51 to 53 , followed by a more gentle slope. This change, particularly for the ${ }_{53} \mathrm{I}$ and ${ }_{55} \mathrm{Cs}$ nuclides is caused by the depression of the first $5 / 2^{+}$level through an admixture with an adjacent $5 / 2^{+}$level arising from the $\left(g_{7 / 2}\right)_{5 / 2+}^{3}$ configuration. The presence and character of such states was described by Paar [27], Heyde [28]; and has also been discussed by Jackson et al., [29] who noted that in closed-shell nuclei this cluster state typically lies at about $1 / 2$ the energy of the $2^{+}$level in the adjacent eveneven core nuclide. The mixing between this cluster state and the single-particle $d_{5 / 2}$ proton state could account for an unexpectedly low position of the lowest $5 / 2^{+}$level in these $N$ $=82$ isotones.

For the $N=78$ and 80 isotones, with more collective cores and lower $2^{+}$energies, a similar pair of $5 / 2^{+}$levels are observed, but at significantly lower energies. However, in both cases, a straight extrapolation as the Fermi level rises from

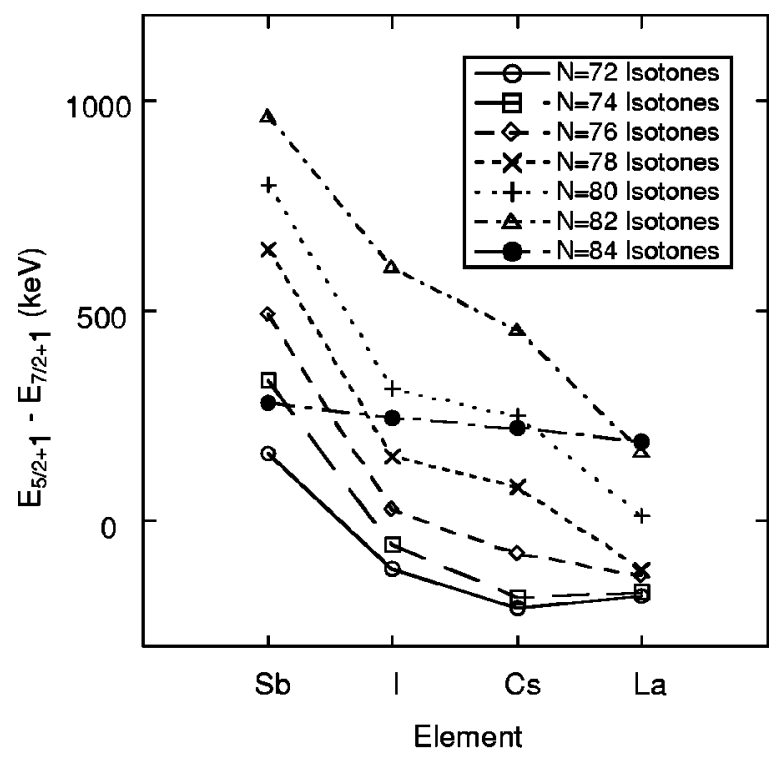

FIG. 13. Variation of the energy difference between the lowest $5 / 2^{+}$and $7 / 2^{+}$levels in the $N=72-84$ even-odd $\mathrm{Sb}, \mathrm{I}, \mathrm{Cs}$, and La isotones. Lines are drawn to guide the eye. 


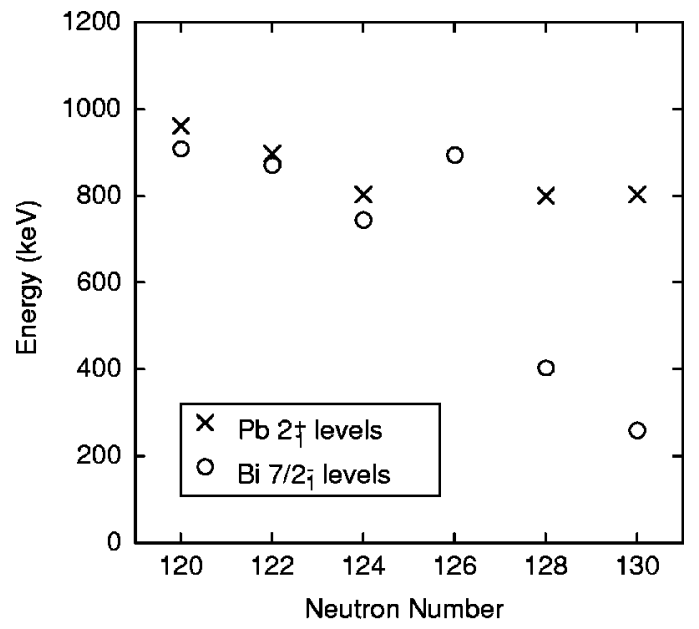

FIG. 14. Comparison of the first $7 / 2^{-}$level energies of Bi nuclei in the region of double-magic ${ }^{208} \mathrm{~Pb}$ with the positions of the $2^{+}$ levels in their corresponding $\mathrm{Pb}$ cores.

$Z=57$ to 55 to 53 to the $Z=51 \mathrm{Sb}$ nuclides would suggest a lower position for the first $5 / 2^{+}$level than is actually observed. Stated another way, an upward jog is to be expected for the position of the lowest $5 / 2^{+}$level in going from the $Z=53$ odd-mass iodine nuclides to the $Z=51 \mathrm{Sb}$ nuclides because of the inability of the single-proton $\mathrm{Sb}$ nuclides to form the three-proton cluster states present in the I and Cs nuclides.

Keeping in mind the important role of the cluster states in depressing the lowest $5 / 2^{+}$level in the odd-mass ${ }_{53} \mathrm{I} \mathrm{nu-}$ clides, it is now possible to interpet the rather flat behavior of the lowest $5 / 2^{+}$level in the $N=84$ isotones. The absence of an upward jog in going from ${ }^{137} \mathrm{I}$ to ${ }^{135} \mathrm{Sb}$ is interpreted to arise because the majority of the $d_{5 / 2}$ strength in ${ }^{135} \mathrm{Sb}$ (and also in ${ }^{137} \mathrm{I}$ ) is already well below the energy of the expected $\left(g_{7 / 2}\right)_{5 / 2+}^{3}$ state.

In Fig. 14, we show the positions of the lowest lying $h_{9 / 2}$ ground states and $f_{7 / 2}$ low-energy levels of the odd-mass $\mathrm{Bi}$ nuclides and the positions of the first $2^{+}$levels in the neighboring $\mathrm{Pb}$ core in the region of the double-magic nuclide ${ }^{208} \mathrm{~Pb}$. In these nuclides, the separation between the $h_{9 / 2}$ ground states and the lower-spin $f_{7 / 2}$ first excited states is observed to be relatively constant below the $N=126$ shell closure, but then to narrow as neutrons are added beyond $N=126$ in a fashion quite similar to the observations in the $\mathrm{Sb}$ nuclides.

Earlier, we suggested that this low-lying $5 / 2^{+}$state arises from a strong admixture of the $d_{5 / 2}$ single-particle configuration; and that such an admixture would require that the unmixed energy of this single $d_{5 / 2}$ proton level must lie at a much lower position than the $963 \mathrm{keV}$ position observed in ${ }^{133} \mathrm{Sb}$ [3]. Support for such an idea has been presented in a recent paper by Hamamoto et al. [16]. They showed that an increase in the Woods-Saxon surface diffuseness parameter would result in a lowering of low-spin single-particle shell model levels relative to higher-spin levels.

To further investigate the structure of these low-energy states, calculations were carried out in the proton-neutron formalism with the shell-model code OXBASH [17]. The model space consists of a ${ }^{132} \mathrm{Sn}$ closed core with $(Z-50)$ valence protons in the $\left(0 g_{7 / 2}, 1 d_{5 / 2}, 1 d_{3 / 2}, 2 s_{1 / 2}, 0 h_{11 / 2}\right)$ orbitals and $(N-82)$ valence neutrons in the $\left(0 h_{9 / 2}, 1 f_{7 / 2}\right.$, $\left.1 f_{5 / 2}, 2 p_{3 / 2}, 2 p_{1 / 2}, 0 i_{13 / 2}\right)$ orbitals. The single-particle energies are taken from experiment. The observed levels in ${ }^{133} \mathrm{Sb}$ [18] provide the proton single-particle energies -9.66, $-8.70,-7.22$, and $-6.87 \mathrm{MeV}$ for the $0 g_{7 / 2}, 1 d_{5 / 2}, 1 d_{3 / 2}$, and $0 h_{11 / 2}$ orbitals, respectively. The observed levels in ${ }^{133} \mathrm{Sn}$ [19] provide the neutron single-particle energies $-0.89,-2.45,-0.45,-1.60$, and $-0.80 \mathrm{MeV}$ for the $0 h_{9 / 2}$, $1 f_{7 / 2}, 1 f_{5 / 2}, 2 p_{3 / 2}$, and $2 p_{1 / 2}$ orbitals, respectively. The single-particle energy of $0.25 \mathrm{MeV} 0 i_{13 / 2}$ orbital was chosen to give the energy of the $10^{+}$level in ${ }^{134} \mathrm{Sb}$ which has been assigned to the $\left(\pi g_{7 / 2}, \nu i_{13 / 2}\right)$ configuration [20].

The residual two-body interaction is obtained starting with a $G$ matrix derived from the recent CD-Bonn [21] nucleon-nucleon interaction, with ${ }^{132} \mathrm{Sn}$ as a closed shell core. A harmonic oscillator basis was employed for the single-particle wave functions with an oscillator energy $\hbar \omega$ $=7.87 \mathrm{MeV}$. The $G$-matrix elements form in turn the starting point for a perturbative derivation of a shell-model effective interaction. In this work we derive the effective interaction for the above shell-model space by the $\hat{Q}$-box method that includes all so-called nonfolded diagrams through third order in the interaction $G$ and sums up the folded diagrams to infinite order [22,23]. This type of Hamiltonian has been used to describe spectra of tin isotopes from mass number $A=102$ to $A=130$ [24] and the $N=82$ isotones up to $A$ $=146$ [25] with good agreement with data.

The calculated spectrum of ${ }^{134} \mathrm{Sn}$ is compared with experiment in Figs. 15(a),(b). There is excellent agreement between experiment [Fig. 15(a)] and theory [Fig. 15(b)]. The calculated and experimental spectra for ${ }^{135} \mathrm{Sb}$ are shown in Figs. 15(c)-(f). The agreement is good for the yrast states [Fig. 15(f)], and the density of $5 / 2^{+}, 7 / 2^{+}$, and $9 / 2^{+}$proton levels below $1.5 \mathrm{MeV}$ that could be populated in $\beta$-decay are consistent with the number of levels observed in this study. In Figs. 15(d),(e), we show all of the levels with spins and energies up to the $19 / 2^{+}$yrast level; and the remaining levels up to $1.5 \mathrm{MeV}$ that could be populated via $\beta$-decay are indicated by a small dot.

To illustrate the mean-field properties of the singleparticle energies we show in Fig. 16 the proton singleparticle energies obtained from the SKX Skryme interaction [26]. They were calculated for the proton-particle states of the ${ }^{101} \mathrm{Sb}$ to ${ }^{145} \mathrm{Sb}$ isotopes and for the proton-hole states of the ${ }^{191} \mathrm{Tl}$ to ${ }^{207} \mathrm{Tl}$ isotopes. One observes a decrease in the $s$ and $d$ single-particle energies relative to $g_{7 / 2}$ after $N=82$ that can be associated with the neutron skin in the heavy Sn isotopes. The decrease in the $d_{5 / 2}-g_{7 / 2}$ splitting between $N$ $=82$ and $N=84$ of $100 \mathrm{keV}$ is not enough to account for the $300 \mathrm{keV}$ required for the shell-model calculation but at least goes in the right direction. This monopole shift is in principle contained in the $G$-matrix interaction. But since the $G$ matrix is calculated in an oscillator basis, it may not contain the skin effects that are present in the Hartree-Fock (HF) calculation.

Table II shows the single-particle nature of the $7 / 2^{+}$and $5 / 2^{+}$states in terms of the ${ }^{134} \mathrm{Sn}$ to ${ }^{135} \mathrm{Sb}$ proton spectro- 


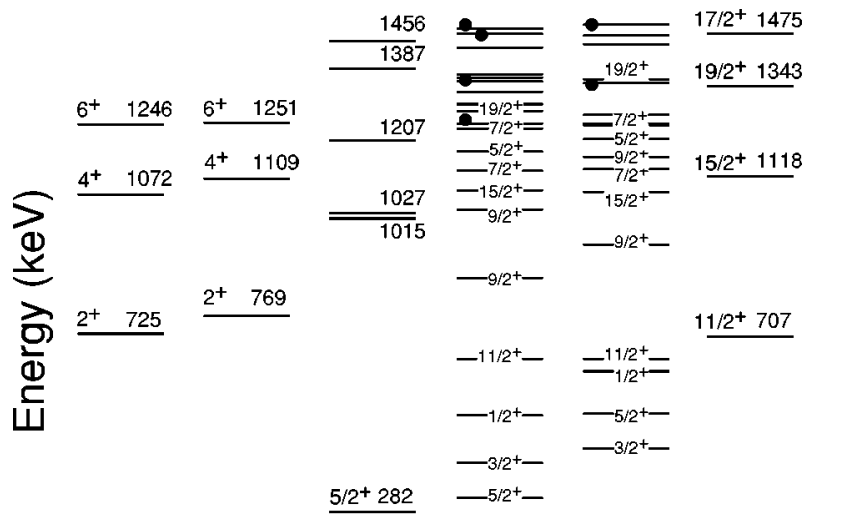

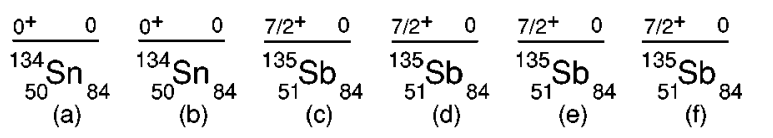

- Denotes a level whose spin and parity is not shown that could be fed by $\beta$-decay of ${ }^{135} \mathrm{Sn}\left(5 / 2^{+}, 7 / 2^{+}\right.$, or $9 / 2^{+}$states $)$

FIG. 15. Comparison of OXBASH calculations and levels reported in this study. In (a) are shown the experimental levels obtained for ${ }^{134} \mathrm{Sn}$ reported by Zhang et al. [20]. In (b), are shown the OXBASH calculations for the corresponding levels for ${ }^{134} \mathrm{Sn}$. The levels obtained for ${ }^{135} \mathrm{Sb}$ in this study are shown in (c), along with other observations reported by Bhattacharyya et al. in (f) [14]. In (d) and (e) are OXBASH calculations for ${ }^{135} \mathrm{Sb}$, with the former being adjusted by lowering the $d_{5 / 2}$ single-particle energy by $300 \mathrm{keV}$.

scopic factors. The centroid of the $g_{7 / 2}$ and $d_{5 / 2}$ singleparticle strengths are -10.54 and $-9.46 \mathrm{MeV}$, respectively. These can be compared with the input single-particle energies of -9.66 and $-8.70 \mathrm{MeV}$, respectively, to observe that $G$-matrix shifts the $d_{5 / 2}$ strength up by about $100 \mathrm{keV}$ relative to $g_{7 / 2}$, rather than the $-100 \mathrm{keV}$ obtained in HF. Thus

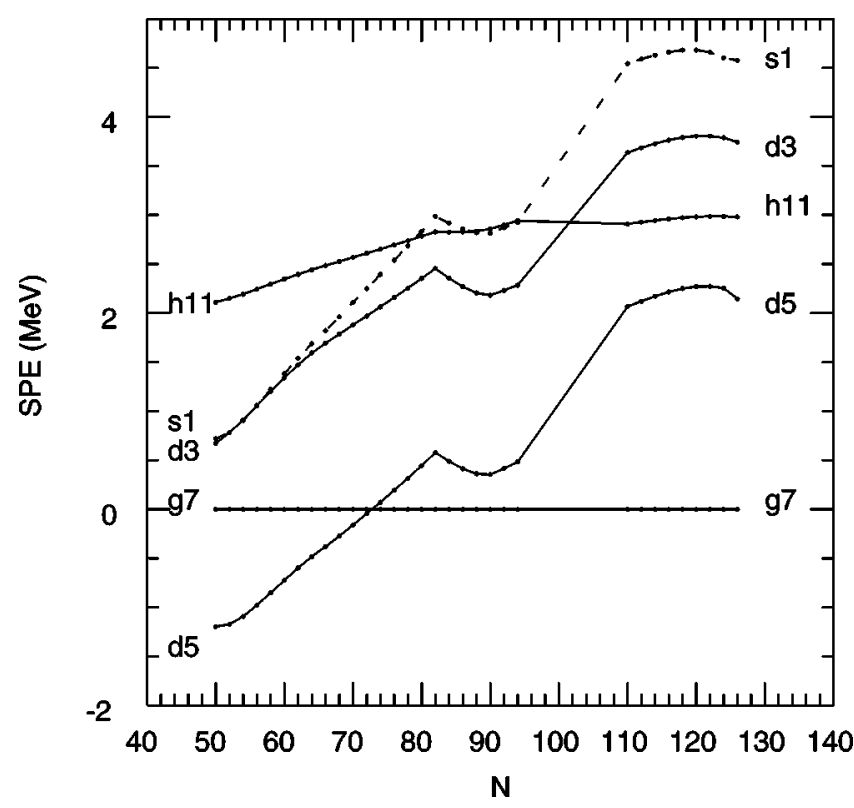

FIG. 16. Proton single-particle energies for odd-even $\mathrm{Sb}$ isotopes with $50 \leqslant N \leqslant 94$ and odd-even $\mathrm{Tl}$ isotopes with $110 \leqslant N$ $\leqslant 128$.
TABLE II. Spectroscopic factors and one-body transition densities.

\begin{tabular}{|c|c|c|c|c|c|}
\hline$J^{\pi}$ & $\begin{array}{c}\mathrm{Ex} \\
(\mathrm{MeV})\end{array}$ & $\mathrm{C}^{2} \mathrm{~S}$ & $\begin{array}{c}\mathrm{OBTD}_{1} \\
\text { rank } 0\end{array}$ & $\begin{array}{c}\mathrm{OBTD}_{1} \\
\text { rank } 1\end{array}$ & $\begin{array}{c}\mathrm{OBTD}_{2} \\
\text { rank 1 }\end{array}$ \\
\hline $7 / 2^{+}$ & 0.00 & 0.692 & 0.304 & 0.550 & 0.071 \\
\hline $7 / 2^{+}$ & 1.14 & 0.186 & -1.489 & -0.649 & -0.029 \\
\hline $7 / 2^{+}$ & 1.25 & 0.008 & 0.346 & -0.455 & -0.015 \\
\hline $7 / 2^{+}$ & 1.57 & 0.000 & 0.051 & -0.346 & -0.059 \\
\hline $7 / 2^{+}$ & 1.59 & 0.079 & 0.087 & 0.280 & -0.411 \\
\hline $7 / 2^{+}$ & 1.65 & 0.015 & -0.118 & -0.026 & -0.116 \\
\hline $7 / 2^{+}$ & other & 0.028 & & & \\
\hline $5 / 2^{+}$ & 0.53 & 0.498 & & -0.261 & -0.432 \\
\hline $5 / 2^{+}$ & 1.21 & 0.098 & & 0.518 & -0.211 \\
\hline $5 / 2^{+}$ & 1.25 & 0.056 & & -0.367 & 0.161 \\
\hline $5 / 2^{+}$ & 1.47 & 0.000 & & -0.125 & -0.012 \\
\hline $5 / 2^{+}$ & 1.89 & 0.018 & & -0.139 & 0.431 \\
\hline $5 / 2^{+}$ & 1.94 & 0.003 & & -0.026 & 0.104 \\
\hline $5 / 2^{+}$ & 2.06 & 0.137 & & -0.121 & -0.263 \\
\hline $5 / 2^{+}$ & 2.18 & 0.031 & & 0.091 & 0.632 \\
\hline $5 / 2^{+}$ & other & 0.159 & & & \\
\hline $9 / 2^{+}$ & 0.95 & & & -0.503 & -0.089 \\
\hline $9 / 2^{+}$ & 1.17 & & & -0.031 & 0.241 \\
\hline $9 / 2^{+}$ & 1.50 & & & -0.693 & 0.126 \\
\hline
\end{tabular}

if we were to combine the shell-model calculation (which takes into account the proton-neutron interaction in an oscillator basis) with the HF calculation, which takes into account the neutron skin, we are able to obtain the energy of the excited $5 / 2^{+}$state (and all other excited states) to within about $100 \mathrm{keV}$.

The splitting of the single-particle strength has a rather complex effect on the first-forbidden $\beta$-decay of ${ }^{135} \mathrm{Sn}$. In our model the $\beta$-decay of ${ }^{133} \mathrm{Sn}$ to the $7 / 2^{+}$and $5 / 2^{+}$states in ${ }^{133} \mathrm{Sb}$ are given by one-body transition densities of $\mathrm{OBTD}_{1}=1$ (rank 0 and 1) and $\mathrm{OBTD}_{2}=1$ (rank 1) for the $f_{7 / 2}$ to $g_{7 / 2}$ and $f_{7 / 2}$ to $d_{5 / 2}$ transitions, respectively. The values of these OBTD for the ${ }^{135} \mathrm{Sn}$ to ${ }^{135} \mathrm{Sb} \beta$-decay are given in Table II (there are many other OBTD which enter at the level of 0.1 or less). The two rank 1 transitions need to be added in phase, thus the implications require a more detailed calculation of the single-particle transition matrix elements. But the implication is that the ${ }^{135} \mathrm{Sn} \beta$-decay will have strong transitions to several states around $1.2 \mathrm{MeV}$ excitation in ${ }^{135} \mathrm{Sb}$ as observed in this experiment.

Note that in the range from 500 to $1250 \mathrm{keV}$ in Fig. 15(e); there are only six states with spins $5 / 2^{+}, 7 / 2^{+}$, and $9 / 2^{+}$. We observe population of three of them. We observe levels at 1015 and $1027 \mathrm{keV}$; and levels are calculated at 947, 1079, and $1135 \mathrm{keV}$. The level at $1207 \mathrm{keV}$ is also quite close to two calculated levels at 1212 and $1246 \mathrm{keV}$. Notice that these features of the calculation are similar in Fig. 15(d). We observe two additional states up to $1.5 \mathrm{MeV}$; whereas, there are only a total of eight or ten states possibly fed as indicated by the two calculations.

Finally we comment on the $E 2$ properties of the nuclei in this region as given in Table III. The $B(E 2)$ were calculated 
TABLE III. Experimental and theoretical $B(E 2)$ values in units of $e^{2} \mathrm{fm}^{4}$.

\begin{tabular}{llcc}
\hline \hline Nucleus & Transition & $\begin{array}{c}B(E 2) \\
\text { experiment }\end{array}$ & $\begin{array}{c}B(E 2) \\
\text { theory }\end{array}$ \\
\hline${ }^{134} \mathrm{Sn}$ & $6^{+}$to $4^{+}$ & $36(7)$ & 41 \\
${ }^{134} \mathrm{Te}$ & $6^{+}$to $4^{+}$ & $83(2)$ & 78 \\
& $4^{+}$to $2^{+}$ & $159(16)$ & 168 \\
& $0^{+}$to $2^{+}$ & & 810 \\
${ }^{136} \mathrm{Te}$ & $0^{+}$to $2^{+}$ & & 2300 \\
\hline \hline
\end{tabular}

with the SKX [26] radial wave functions and effective charges of $e_{p}=1.5$ and $e_{n}=0.7$ obtained from the ${ }^{134} \mathrm{Te}$ and ${ }^{134} \mathrm{Sn} 6^{+}$to $4^{+}$transitions, respectively [20].

\section{SUMMARY}

In this paper, results for the $T_{1 / 2}$ and $P_{n}$ values of the very neutron rich $\mathrm{Sn}$ nuclides, ${ }^{135,136,137} \mathrm{Sn}$ have been presented, along with additional level structure properties for ${ }^{135} \mathrm{Sb}$. These results are already incorporated into codes for calcu- lating $r$-process abundances and also serve as the basis of better estimates for unknown half-lives of critical waitingpoint nuclides such as ${ }^{138} \mathrm{Sn}$. The $282-\mathrm{keV}$ position of the first excited state of ${ }^{135} \mathrm{Sb}$ was found at an unexpectedly low energy. Its low position provides support for the idea that nuclei with an $N / Z$ ratio that exceeds 1.6 have a more diffuse nuclear surface that changes the relative binding energies of low-spin orbitals when compared to higher-spin orbitals. Shell-model calculations provide a level scheme in close agreement with both the observed yrast levels and the levels populated in $\beta$-decay, with the only divergence surrounding the lowest $5 / 2^{+}$level. For that level, the suggested lowering of the single-particle $d_{5 / 2}$ state by $300 \mathrm{keV}$ does provide a better fit for that level without disturbing the otherwise excellent agreement between theory and experiment.

\section{ACKNOWLEDGMENTS}

We are pleased to acknowledge the support of the ISOLDE technical staff during this experiment. Support for this work was provided by the U.S. Department of Energy, the deutsche BMBF under Contract No. 06MZ864, and National Science Foundation under Contract No. PHY0070911.
[1] K.-L. Kratz et al., Nucl. Phys. A688, 308 (2001).

[2] K.-L. Kratz et al., Hyperfine Interact. 129, 185 (2000).

[3] J. Shergur et al., Proceedings of NS2000, MSU [Nucl. Phys. A682, 493 (2001)].

[4] V.I. Mishin et al., Nucl. Instrum. Methods Phys. Res. B 73, 550 (1993).

[5] N. Erdmann et al., Appl. Phys. B: Lasers Opt. 66, 431 (1998).

[6] M. Hannawald et al., Phys. Rev. C 62, 054301 (2000).

[7] M. Hannawald et al., Phys. Lett. 82, 1391 (1999).

[8] B. Pfeiffer et al., Progr. Nucl. Energ. (to be published), www.arXiv.org/abs/nucl-ex/0106020.

[9] A. Korgul et al., Phys. Rev. C 64, 021302 (2001).

[10] B. Fogelberg et al., Phys. Rev. C 41, R1890 (1990).

[11] P. Hoff et al., Z. Phys. A 332, 407 (1989).

[12] M.N. Mineva, Eur. Phys. J. A 11, 9 (2001).

[13] K.-L. Kratz et al., Revs. Mod. Astr. 1, 184 (1988).

[14] P. Bhattacharyya et al., Eur. Phys. J. A 3, 109 (1998).
[15] M. Sanchez-Vega et al., Phys. Rev. C 60, 024303 (1999).

[16] I. Hamamoto et al., Nucl. Phys. A683, 255 (2001).

[17] A. Etchegoyen et al., MSU-NSCL Report No. 524, 1985.

[18] M. Sanchez-Vega et al., Phys. Rev. Lett. 80, 5504 (1998).

[19] P. Hoff et al., Phys. Rev. Lett. 77, 1020 (1996).

[20] C.T. Zhang et al., Z. Phys. A: Hadrons Nucl. 358, 9 (1997).

[21] R. Machleidt et al., Phys. Rev. C 53, R1483 (1996).

[22] M. Hjorth-Jensen et al., Phys. Rep. 261, 125 (1995).

[23] M. Hjorth-Jensen et al., J. Phys. G 22, 321 (1996).

[24] A. Holt et al., Nucl. Phys. A634, 41 (1998).

[25] A. Holt et al., Nucl. Phys. A618, 107 (1997).

[26] B.A. Brown, Phys. Rev. C 58, 220 (1998).

[27] V. Paar et al., Nucl. Phys. A211, 29 (1973).

[28] K. Heyde, The Nuclear Shell Model (Springer-Verlag, New York, 1994), p. 121.

[29] S.V. Jackson et al., Phys. Rev. C 11, 1323 (1975). 